To be submitted to Geophysical Research Letters, 1998.

\title{
Kinetic Ballooning Instability for Substorm Onset and Current Disruption Observed by AMPTE/CCE
}

\author{
C. Z. Cheng \\ Princeton Plasma Physics Laboratory, Princeton University, Princeton, NJ 08543
}

A. T. Y. Lui

Applied Physics Laboratory, Johns Hopkins University, Laurel, MD 20723

\begin{abstract}
A new scenario of AMPTE/CCE observation of substorm onset and current disruption and the corresponding physical processes is presented. Toward the end of late growth phase plasma $\beta$ increases to $\geq 50$ and a low frequency instability with a wave period of $50-75$ seconds is excited and grows exponentially to a large amplitude at the onset of current disruption. At the current disruption onset higher frequency instabilities are excited so that the plasma and electromagnetic field form a turbulent state. Plasma transport takes place to modify the ambient plasma pressure and velocity profiles so that the ambient magnetic field recovers from a tail-like geometry to a more dipole-like geometry. To understand the excitation of the low frequency global instability, a new theory of kinetic ballooning instability (KBI) is proposed to explain the high critical $\beta$ threshold $\left(\beta_{c} \geq 50\right)$ of the low frequency global instability observed by the AMPTE/CCE. The stabilization is mainly due to kinetic effects of trapped electrons and finite ion Larmor radii which give rise to a large parallel electric field and hence a parallel current that greatly enhances the stabilizing effect of field line tension to the ballooning mode. As a result $\beta_{c}$ for excitation of KBI is greatly increased over the ideal MHD ballooning instability threshold by $\geq O\left(10^{2}\right)$. The wave-ion magnetic drift resonance effect produces a perturbed resonant ion velocity distribution with a duskward velocity roughly equal to the average ion magnetic ( $\nabla B$ and curvature) drift velocity. Higher frequency instabilities such as cross-field current instability (CCI) can be excited by the additional velocity space free energy associated with the positive slope in the perturbed resonant ion velocity distribution in the current disruption phase.
\end{abstract}

\section{Introduction}

A critical process in the previously established view of the substorm onset and current disruption based on the observation of AMPTE/CCE spacecraft is the explosive growth phase (which lasts $\sim 30$ seconds) at the approach of current disruption onset [Ohtani et al., 1992]. During the explosive growth phase a large upsurge in the duskward ion bulk drift to nearly the ion thermal velocity is found near the local midnight sector which could lead to the excitation of the cross-field current instability (CCI) during the cross tail current disruption phase [Lui, 1996]. In this paper a new scenario of the AMPTE/CCE observation of substorm explosive growth phase, onset and current disruption is presented. In particular, toward the end of late growth phase (approximately 2 minutes before the onset of current disruption) plasma pressure be- 
comes isotropic and $\beta$ increases to $\geq 50$ and we have found that a low frequency global instability with a wave period of $\sim 50-75$ seconds is excited and grows exponentially to a large amplitude with $\delta B / B \geq 0.3$ at the onset of current disruption. The half wave period of the instability before the current disruption onset corresponds to the explosive growth phase. At the current disruption onset higher frequency instabilities (with wave periods $15 \mathrm{sec}, 10$, sec, $5 \mathrm{sec}$, etc.) are excited and the plasma and electromagnetic field form a strong turbulent state for $\sim 4-5$ minutes. During the strong turbulent state anomalously fast plasma transport and heating take place to modify the average plasma pressure and flow profiles so that the ambient magnetic field recovers from a tail-like geometry to a more dipole-like geometry.

Two key issues need to be resolved in order to further understand the physical processes of the current disruption and subsequent magnetic field dipolarization: (1) the excitation mechanism and the high plasma $\beta$ threshold ( $\geq 50$ ) of the low frequency global instability that underlines the explosive growth phase; (2) the physical mechanism of the enhanced ion drift in the explosive growth phase that leads to excitation of higher frequency instabilities. To understand these two key issues, we have developed a new theory of kinetic ballooning instability (KBI), which results from the release of configuration space ballooning free energy of nonuniform pressure with gradient in the same direction as the magnetic field curvature. Previously ballooning instability based on ideal MHD model has been proposed to explain the current disruption [Roux et al., 1991]. Ideal MHD ballooning mode theory would predict a low $\beta_{c}(\leq 1)$ for instability, which is contrary to the high $\beta$ values $(\geq 20)$ observed by AMPTE/CCE throughout the late growth phase. In this paper we show that kinetic effects such as trapped particle dynamics, finite ion Larmor radii (FLR) and wave-particle resonances are important in determining the stability of KBI and we are able to answer these two key issues of substorm onset and current disruption.

The kinetic ballooning instability theory properly explains the wave frequency, growth rate and high $\beta_{c}(>50)$ of the low frequency instability observed by the AMPTE/CCE. Because the wave phase velocity along the field line is smaller than the electron thermal velocity, the trapped electron effect coupled with ion FLR effects causes a large parallel electric field and thus a much enhanced parallel current which greatly enhances the stabilizing field line tension over the value expected from the MHD theory. As a result, a much higher $\beta_{c}$ than that based on the ideal MHD model is obtained with $\beta_{c} \geq O\left(10^{2}\right) \beta_{c}^{M H D}$, where $\beta_{c}^{M H D}$ is the critical $\beta$ predicted by the MHD theory. The effect of the wave-ion magnetic drift resonance with $\omega-\omega_{d i}=0$, where $\omega_{d i}$ is the ion magnetic drift frequency, provides an additional channel to release the free energy and typically reduces $\beta_{c}$ by up to $20 \%$.

Another consequence of the wave-particle resonance is to produce a localized perturbed ion distribution in the velocity space centered around $v_{y}=v_{d i}$, where $v_{y}$ is the particle velocity in the dusk direction and $v_{d i}$ is the average ion magnetic $(\nabla B$ and curvature) drift velocity. As KBI grows to a large amplitude, the perturbed ion velocity distribution increases so that $\partial f_{i} / \partial v_{y}>0$ for $v_{y} \leq v_{d i}$, which provides an additional free energy source for higher frequency instabilities such as CCI. As the higher frequency instabilities quickly grow to large amplitudes, they combine with KBI to form a strongly plasma turbulence, which leads to anomalously large plasma transport and heating in the current disruption phase. In a few minutes the plasma $\beta$ decreases and the pressure profile relaxes to a more quiet time-like profile and the magnetic field recovers to a more dipole-like geometry. Thus, the new substorm scenario emphasizes a global low-frequency mode (KBI) which can naturally account for the explosive growth phase and the initiation of subsequent current disruption through a combination of KBI and CCI.

\section{Low Frequency Global Instability Ob- served by AMPTE/CCE}

Evidence for the low-frequency perturbations occurring prior to current disruption onset can be found in detailed examination of magnetic field during current disruption events. Figure 1 shows the result of such an analysis. Magnetic field measurements from AMPTE/CCE on August 30, 1986 were studied. The satellite was in the midnight local time sector, MLT $=$ 23.5 , at a radial distance of approximately $8 R_{E}$. The current disruption onset time is determined to be at 11:52:40 UT [Takahashi et al., 1987; Lui et al., 1992; Ohtani et al., 1995]. To extract the low frequency components of the fluctuations, we employed successive smoothing of the original signals with normalized binomial coefficients as previously used in [Lui and Najmi, 1997]. The derived magnetic field perturbations in the cylindrical $(V, D, H)$ components were then converted into components in the unperturbed 


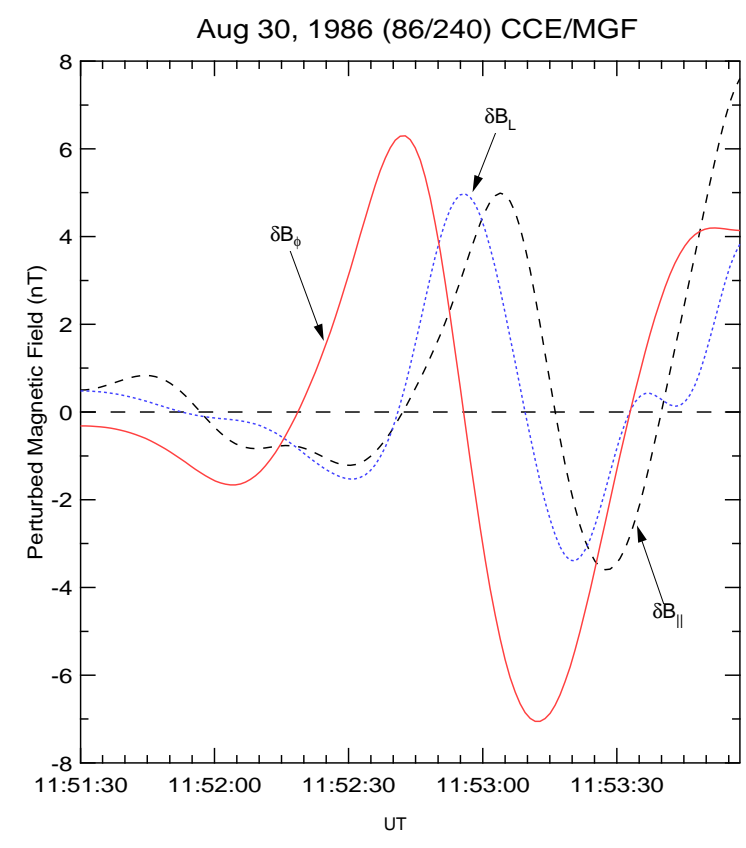

Figure 1. Three components of the low frequency perturbed magnetic field.

magnetic field coordinate system to give $\delta B_{L}, \delta B_{\phi}$, and $\delta B_{\|}$, where positive $\delta B_{\phi}$ is pointing eastward, positive $\delta B_{\|}$is along the mean magnetic field direction and positive $\delta B_{L}$ is in the third right-handed orthogonal direction. From Figure 1, one can deduce that the real frequency of the low-frequency perturbation is $\sim 0.1 \mathrm{~Hz}$ (compared with proton cyclotron frequency of about $1 \mathrm{~Hz}$ ) and the growth rate is about 0.2 of the real frequency. Note that the exponentially low-frequency perturbation begins quite early at $\sim 11: 51: 30 \mathrm{UT}, \sim 1.5 \mathrm{~min}$ before the onset of current disruption. It is interesting to note that there is almost no magnetic field fluctuation before the low frequency global instability was observed. Although not shown here, the general characteristics of the low frequency perturbation given here are also found in another current disruption event (June 1, 1985) [Lui et al., 1992] which we have examined.

The $30 \mathrm{sec}$ exponential growth phase period of the low frequency global instability (with amplitude reaching $\delta B / B \geq 0.3)$ just before the current disruption onset was previously called "explosive growth phase" [Ohtani et al., 1992] which is accompanied by a large duskward shift of the ion velocity distribution function and hence a significantly enhanced cross-tail current density [Lui, 1996]. This enhanced cross-tail ion drift population is responsible for exciting higher frequency instabilities such as CCI which together with the low frequency global instability last through out the current disruption phase and form a strong magnetic field turbulence.

During the late growth phase of substorms the plasma pressure in the midnight sector of the nearEarth tail $\left(\sim 7-9 \mathrm{R}_{\mathrm{E}}\right)$ observed by the AMPTE/CCE [Lui et al., 1992] usually increases $\sim 50 \%$ from $\sim 10$ minutes prior to the current disruption onset to the time the low frequency global instability is observed. The corresponding plasma $\beta$ usually increases from $\sim 10$ to $\geq 60$. This pressure change leads to crosstail current enhancement and thinning of the plasma sheet. Although there is no observational determination on the pressure profile during the late growth phase, it is reasonable to expect that the plasma pressure decreases monotonically with increasing radial distance. Energy associated with the current enhancement can be viewed as being stored in the largescale magnetic field on the nightside.

\section{Kinetic Ballooning Instability}

Based on the AMPTE/CCE observation of particle data [Lui et al., 1992; Lui, 1996], at the end of the growth phase the average electron energy is about $5 \mathrm{keV}$ and the average ion energy is about $10 \mathrm{keV}$. The particle velocity distribution function does not have appreciable bulk drift and the plasma pressure becomes isotropic in the late growth phase in a few minutes before the onset. The average ion magnetic drift velocity evaluated at the average ion energy is about the same as the ion thermal velocity if the $\nabla B$ scale length is on the order of average ion Larmor radius. With these information we consider KBI perturbations with the orderings: $k_{\perp} \rho_{i} \sim O(1)$ and $k_{\|} \ll k_{\perp}$ and $v_{\text {the }}>\left(\omega / k_{\|}\right)>v_{\text {thi }}[$ Cheng, 1982b, $1982 \mathrm{a}$, where the subscripts $\|$ and $\perp$ represent parallel and perpendicular components to the equilibrium magnetic field. With these orderings the following kinetic effects must be considered: trapped electron dynamics, ion FLR effect and wave-particle resonance with $\omega-\omega_{d i}=0$. We shall obtain approximate solutions of the perturbed particle distributions based on the gyrokinetic formulation by assuming that both electrons and ions have local Maxwellian equilibrium distribution functions [Cheng et al., 1995].

We shall consider a three-dimensional magnetospheric equilibrium with the magnetic field expressed as $\mathbf{B}=\nabla \psi \times \nabla \alpha$, where $\psi$ is chosen as the mag- 
netic flux function which is a function of $L$-shell only and $\alpha$ is an azimuthal-angle like variable with a period of $2 \pi$. The guiding center particle equilibrium distribution is assumed to be $F=F(\mathcal{E}, \psi)$ so that the equilibrium pressure is a function of $\psi$ only. We consider perturbations with $k_{\|} L_{\|}>1$ and $k_{\perp} L_{\perp}>1$, and $L_{\|}>L_{\perp}$, where $L_{\|, \perp}$ are the parallel and perpendicular background equilibrium scale lengths respectively. We assume a WKB eikonal representation for perturbed quantities, i.e., $\delta f(\vec{x}, \vec{v}, t)=\delta f\left(s, \mathbf{k}_{\perp}, \vec{v}, t\right) \exp \left[i\left(\int d \vec{x}_{\perp} \cdot \mathbf{k}_{\perp}-\omega t\right)\right]$. Including full FLR effects the perturbed particle distribution function can be expressed in terms of the rationalized MKS unit as $\delta f=(q / M) \partial F / \partial \mathcal{E}\left[\left(\omega_{\star}^{T} / \omega\right) \Phi-\right.$ $\left(1-\omega_{\star}^{T} / \omega\right)\left(1-J_{0} e^{i \delta L}\right) \Phi+g e^{i \delta L}$, where $M$ is the particle mass, $q$ is the particle charge, $B$ is the magnetic field intensity, $\delta L=\mathbf{k} \times \mathbf{v} \cdot \hat{\mathbf{b}} / \omega_{c}, J_{l}$ is the $l$ th order Bessel function of the argument $k_{\perp} v_{\perp} / \omega_{c}$, $\omega_{c}=q B / M$ is the cyclotron frequency, $\Phi$ is the perturbed electrostatic potential, and $g$ is the nonadiabatic part of the perturbed distribution function. Based on the WKB-ballooning formalism the lowest order gyrokinetic equation for $g$ in the low frequency $\left(\omega \ll \omega_{c}\right)$ limit is given by

$$
\begin{gathered}
\left(\omega-\omega_{d}+i \mathbf{v}_{\|} \cdot \nabla_{\|}\right) g=-\frac{q}{M} \frac{\partial F}{\partial \mathcal{E}}\left(1-\frac{\omega_{\star}^{T}}{\omega}\right) \\
\times\left[\left(\omega_{d} \Phi-i \mathbf{v}_{\|} \cdot \nabla_{\|} \Psi\right) J_{0}+\frac{\omega v_{\perp}}{k_{\perp}} J_{1} \delta B_{\|}\right],
\end{gathered}
$$

where $\Psi$ is the parallel perturbed electric field potential with $\mathbf{E}_{\|}=-\nabla_{\|} \Psi, \delta B_{\|}$is the parallel perturbed magnetic field, $\omega_{\star}^{T}=\mathbf{B} \times \mathbf{k}_{\perp} \cdot \nabla F /\left(B \omega_{c} \partial F / \partial \mathcal{E}\right), \omega_{d}=$ $\mathbf{k}_{\perp} \cdot \mathbf{v}_{d}$ is the magnetic drift frequency, $\mathbf{v}_{d}=\left(\mathbf{B} / B \omega_{c}\right) \times$ $\left(v_{\|}^{2} \boldsymbol{\kappa}+\mu \nabla B\right)$ is the magnetic drift velocity, and $\boldsymbol{\kappa}$ is the magnetic field curvature. Note that the vector potential, defined by $\mathbf{A}=\mathbf{A}_{\|}-i A_{\perp} \mathbf{B} \times \mathbf{k}_{\perp} /\left(B k_{\perp}\right)$, is related to $\Phi, \Psi$ and $\delta B_{\|}$by $\omega \mathbf{A}_{\|}=-i \nabla_{\|}(\Phi-\Psi)$ and $\delta B_{\|}=k_{\perp} A_{\perp}$. We note that the gyrokinetic formulation is still valid for the case $\rho_{i} \sim L_{\perp}$ if the the magnetic drift frequency is replaced with the pitch angle average value to account for the non-conservation of the magnetic moment [Hurricane et al., 1994].

For electrons we shall neglect FLR effects and consider $\left|v_{\|} \nabla_{\|}\right| \gg \omega, \omega_{d e}$. Clearly, trapped and untrapped electrons have very different parallel dynamics. The un-trapped electron dynamics is mainly determined by its fast parallel transit motion, and to the lowest order in $\left(\omega /\left|v_{\|} \nabla_{\|}\right|\right)$the perturbed un-trapped electron density is given by [Cheng, 1982a]

$$
\delta n_{e u}=\frac{e N_{e u}}{T_{e}}\left[\frac{\omega_{* e}}{\omega} \Phi+\left(1-\frac{\omega_{* e}}{\omega}\right) \Psi\right],
$$

where $\omega_{* e}=\mathbf{B} \times \nabla N_{e} \cdot \mathbf{k}_{\perp} /\left(B \omega_{c e} N_{e}\right)$ is the electron diamagnetic drift frequency, $N_{e u} / N_{e}=1-$ $\left[1-B(s) / B_{i}\right]^{1 / 2}$ is the fraction of un-trapped electron at the field line location $s, B_{i}$ is the magnetic field at the ionosphere. Near the equator $N_{e u} / N_{e} \simeq$ $B(s) / 2 B_{i} \ll 1$.

The trapped electron dynamics is mainly determined by its fast parallel bounce motion and to the lowest order in $\left(\omega / \omega_{b e}\right)$

$$
\delta n_{e t} \simeq \frac{e N_{e t}}{T_{e}}\left[\frac{\omega_{* e}}{\omega} \Phi+\left(1-\frac{\omega_{* e}}{\omega}\right) \Delta \Psi\right]+\delta \hat{n}_{e t},
$$

where $N_{e t} / N_{e}=\left[1-B(s) / B_{i}\right]^{1 / 2}$ is the fraction of trapped electron,

$$
\Delta=\int_{t r} d^{3} v\left(F_{e} / N_{e t}\right)\left[1-\frac{\left\langle\left(\omega-\omega_{d e}\right) \Psi\right\rangle}{\left\langle\omega-\omega_{d e}\right\rangle \Psi}\right],
$$

$\delta \hat{n}_{e t}=-\int_{t r} d^{3} v\left(e F_{e} / T_{e}\right)\left[\left(\omega-\omega_{\star e}^{T}\right) /\left(\omega-\left\langle\omega_{d e}\right\rangle\right)\right]$ $\left\langle\left(\omega_{d e} / \omega\right) \Phi+v_{\perp}^{2} \delta B_{\|} / 2 \omega_{c e}\right\rangle$, and $\left\langle\omega_{d e}\right\rangle$ is the trapped particle orbit average of $\omega_{d e}$. Note that $\Delta \ll 1$ near the equator.

To obtain perturbed ion distribution function we assume that $\omega, \omega_{d i} \gg\left|v_{\|} \nabla_{\|}\right|$, and the nonadiabatic perturbed distribution function is given by

$$
g_{i} \simeq \frac{e F_{i}}{T_{i}} \frac{\omega-\omega_{\star i}^{T}}{\omega-\omega_{d i}}\left(\frac{\omega_{d i} J_{0} \Phi}{\omega}+\frac{v_{\perp} J_{1} \delta B_{\|}}{k_{\perp}}\right) .
$$

Note that the ion dynamics is mainly determined by its perpendicular motion and the perturbed ion density is given by

$\delta n_{i}=-\frac{e N_{i}}{T_{i}}\left[\frac{\omega_{* i}}{\omega} \Phi+\left(1-\frac{\omega_{* p i}}{\omega}\right)(1-\Gamma) \Phi\right]+\delta \hat{n}_{i}$,

where $\omega_{* i}=\mathbf{B} \times \nabla N_{i} \cdot \mathbf{k}_{\perp} /\left(B \omega_{c i} N_{i}\right), \omega_{* p i}=\mathbf{B} \times$ $\nabla P_{i} \cdot \mathbf{k}_{\perp} /\left(B \omega_{c i} P_{i}\right), \Gamma\left(b_{i}\right)=I_{0}\left(b_{i}\right) \exp \left(-b_{i}\right), b_{i}=$ $k_{\perp}^{2} T_{i} / M_{i} \omega_{c i}^{2}=k_{\perp}^{2} \rho_{i}^{2} / 2, I_{0}$ is the modified Bessel function of the zeroth order, and $\delta \hat{n}_{i}=\int d^{3} v g_{i} J_{0}$.

From the charge quasi-neutrality condition we obtain the parallel electric field potential

$$
\begin{array}{r}
\left(\frac{N_{e u}+N_{e t} \Delta}{N_{e}}\right) \Psi=-\frac{T_{e}}{T_{i}} \frac{\omega-\omega_{* p i}}{\omega-\omega_{* e}}(1-\Gamma) \Phi \\
+\frac{T_{e}}{e N_{e}}\left(\delta \hat{n}_{i}-\delta \hat{n}_{e}\right)
\end{array}
$$

In comparison with the limit without trapped electron effects, the parallel electric field is enhanced by $N_{e} /\left(N_{e u}+N_{e t} \Delta\right)$ which is much larger than unity near the equator for $b_{i} \sim O(1)$. Making use of the 
parallel Ampere's law the perturbed parallel current is given by $\delta J_{\|} \simeq i \nabla_{\perp}^{2} \nabla_{\|}(\Phi-\Psi) / \omega$, which represents the enhancement of stabilizing field line tension due to effects of trapped electrons and ion FLR.

To obtain the eigenmode equation for ballooning instability we follow the derivation presented in the paper by Cheng et al. [1995]. By multiplying the gyrokinetic equation with particle charge, integrating it over the velocity space and summing it over all species, and making use of the parallel component of the Ampere's law we obtain

$$
\begin{gathered}
\mathbf{B} \cdot \nabla\left[\frac{k_{\perp}^{2}}{B^{2}} \mathbf{B} \cdot \nabla(\Phi-\Psi)\right]+\frac{\omega\left(\omega-\omega_{* p i}\right)}{V_{A}^{2}} \frac{1-\Gamma\left(b_{i}\right)}{\rho_{i}^{2} / 2} \Phi \\
+\frac{\mathbf{B} \times \boldsymbol{\kappa} \cdot \mathbf{k}_{\perp}}{B^{2}}\left(\frac{2 \mathbf{B} \times \nabla P \cdot \mathbf{k}_{\perp}}{B^{2}} \Phi-\omega \sum_{j} \delta \hat{p}_{j}\right)=0
\end{gathered}
$$

where $V_{A}=B /\left(n_{i} M_{i}\right)^{1 / 2}$ is the Alfvén speed, and the nonadiabatic perturbed pressures for each particle species are given by $\delta \hat{p}_{j}=M_{j} \int d^{3} v\left[\left(1-\omega_{\star j}^{T} / \omega\right)(1-\right.$ $\left.\left.J_{0}^{2}\right) \Phi+g_{j} J_{0}\right]\left(v_{\perp}^{2} / 2+v_{\|}^{2}\right)$. Note that the relation $\mathbf{B}$. $\delta \mathbf{B}+\delta P_{\perp} \simeq 0$ is used for low frequency instabilities with $\omega \ll k_{\perp} V_{A}$ [Cheng, 1991; Cheng and Qian, 1994; Cheng et al., 1995].

Equations (7) and (8) form a coupled set of kinetic ballooning eigenmode equations for solving $\Phi$ and $\Psi$ along the field lines and the eigenvalue $\omega$. We also need to obtain the nonadiabatic contributions of perturbed electron density, $\delta \hat{n}_{e t}$ and $\delta \hat{n}_{i}$, and perturbed particle pressures, $\delta \hat{p}_{\|}$and $\delta \hat{p}_{\perp}$. The eigenmode equations include kinetic effects of trapped electron dynamics, parallel electric field, full ion FLR, and wave-particle resonances. Considering the ordering $\omega \gg \omega_{d e}, \omega_{d i}$, the nonadiabatic density and pressure responses in Eqs. (7) and (8) can be neglected and we obtain a kinetic ballooning mode equation that retains the trapped electron and ion FLR effects, and the local dispersion relation for KBI is approximately given by

$$
\frac{\omega\left(\omega-\omega_{* p i}\right)}{\left(1+b_{i}\right) V_{A}^{2}} \simeq S k_{\|}^{2}-\frac{2 \boldsymbol{\kappa} \cdot \nabla P}{B^{2}},
$$

where $S=1+\left(b_{i} /\left(1+b_{i}\right)\right) N_{e} T_{e} /\left(N_{e u}+N_{e t} \Delta\right) T_{i} \gg 1$, and we have adopted the Padé approximation $1-\Gamma \simeq$ $b_{i} /\left(1+b_{i}\right)$. The real frequency of KBI is $\omega_{r}=\omega_{* p i} / 2$ and the critical $\beta$ is given by

$$
\beta_{c} \simeq S \beta_{c}^{M H D}+\frac{\omega_{* p i}^{2} R_{c} L_{p}}{4\left(1+b_{i}\right) V_{A}^{2}}
$$

where $R_{c}$ is the radius of the magnetic field curvature and $L_{p}$ is the pressure gradient scale length, and $\beta_{c}^{M H D}=k_{\|}^{2} R_{c} L_{p}$ is the ballooning instability threshold based on the MHD theory. It has also previously been calculated that $\beta_{c}^{M H D} \simeq 0.2$ for a dipole field with $L_{p}=1 R_{E}$ at $L=8$ [Cheng and Qian, 1994]. For a more tail-like field we expect that $\beta_{c}^{M H D}$ will be even smaller. Note that based on the magnetic field and plasma parameters observed by AMPTE/CCE: $B=10 n T, T_{e} / T_{i}=0.5, b_{i}=0.5$, we have $S \simeq 10^{2}-10^{3}$, then $\beta_{c} \geq O\left(10^{2}\right) \beta_{c}^{M H D} \sim O(10)$, which is consistent with the AMPTE/CCE observation.

If $\omega \sim \omega_{d i}$, the wave-ion magnetic drift resonance can modify the growth rate and critical $\beta$. To fully evaluate the effect of wave-ion magnetic drift resonance, we need to retain ion nonadiabatic responses in perturbed density and pressures. Numerical studies of KBI have been performed for tokamaks previously [Cheng, 1982b, 1982a] and the results indicated that the effect of the wave-ion magnetic drift resonance is to reduce $\beta_{c}$ by at most $20 \%$ and the real frequency of KBI will increase to $\omega_{* p i}$ at critical $\beta$. We expect the results for the magnetosphere will be qualitatively similar to the tokamak case and the detailed numerical solutions will be presented in the future.

One consequence of the wave-ion magnetic drift resonance is that as KBI grows to a large value with $\delta B / B \geq 0.3$ the perturbed resonant ion velocity distribution has a positive slope near the duskward resonant ion magnetic drift velocity. This can be clearly seen from the $\left(\omega-\omega_{d i}\right)$ resonance denominator in the perturbed ion distribution. Because $\omega_{r} \simeq \omega_{* p i} / 2$, the wave-ion magnetic drift resonance will occur at $v_{d i}=T_{i} \mathbf{B} \times \nabla P_{i} /\left(2 e P_{i} B^{2}\right) \simeq v_{t h i} \rho_{i} / 2 L_{p i}$, where $\rho_{i}$ is the ion Larmor radius and $L_{p i}$ is the ion pressure gradient scale length. Thus, $\left|v_{d i}\right| \sim v_{t h i}$ for $\rho_{i} \sim L_{p i}$.

\section{Summary and Discussion}

In this paper we have identified a new scenario and physical processes of substorm onset and current disruption observed by AMPTE/CCE. Approximately 2 minutes before the current disruption onset, a global kinetic ballooning instability with period of about $50-75$ seconds is excited as the plasma $\beta$ increases above a high threshold value of $\geq 50$. The plasma $\beta$ in the growth phase is usually larger than 10 which is much larger than the ballooning mode $\beta$ threshold based on the ideal MHD theory. As KBI grows to a large amplitude with $\delta B / B>0.3$, the wave-ion 
resonance with $\omega-\omega_{d i}=0$ produces a perturbed ion distribution centered around $v_{y} \sim v_{d i}$ and thus the full ion distribution function has a positive slope with $\partial F_{i} / \partial v>0$ for $v_{y} \leq v_{d i}$. The perturbed ion distribution provides the velocity space free energy to excite instabilities such as CCI. As these low and high frequency instabilities grow, a strong plasma turbulence can be developed fully to yield large plasma transport (via diffusion, convection as well as magnetic reconnection) and heating. In a few minutes the plasma pressure profile averaged over the fast fluctuation time scales is changed so that the magnetic field recovers to a more dipole-like geometry that satisfies the force balance equation.

Acknowledgments This work is supported by the NSF Grants No. ATM-9523331 and No. ATM9622080 and the DoE Contract No. DE-AC02-76CHO3073.

\section{References}

Cheng, C. Z., High-n collisionless ballooning modes in axisymmetric toroidal plasmas, Nucl. Fusion, 22, 773-781, 1982a.

Cheng, C. Z., Kinetic theory of collisionless ballooning modes, Phys. Fluids, 25, 1020-1026, 1982b.

Cheng, C. Z., A kinetic-magnetohydrodynamic model for low-frequency phenomena, J. Geophys. Res., 96, 21,159-21,171, 1991.

Cheng, C. Z., and Q. Qian, Theory of ballooningmirror instabilities for anisotropic pressure plasmas in the magnetosphere, J. Geophys. Res., 99, 11,193-11,209, 1994.

Cheng, C. Z., N. N. Gorelenkov, and C. T. Hsu, Fast particle destabilization of TAE modes, Nucl. Fusion, 35, 1639-1650, 1995.
Hurricane, O. A., R. Pellat, and F. V. Coroniti, The kinetic response of a stochastic plasma to low frequency perturbations, Geophys. Res. Lett, 21, 253, 1994.

Lui, A. T. Y., Current disruption in theEarth's magnetosphere: Observations and models, J. Geophys. Res., 101, 13067, 1996.

Lui, A. T. Y., and A.-H. Najmi, Time-frequency decomposition of signals in a current disruption event, Geophys. Res. Lett., 24, 3157, 1997.

Lui, A. T. Y., et al., Current disruptions in the nearEarth neutral sheet region, J. Geophys. Res., 97, 1461, 1992.

Ohtani, S., K. Takahashi, L. Zanetti, T. A. Potemra, R. W. McEntire, and T. Iijima, Initial signatures of magnetic field and energetic particle fluxes at tail reconfiguration: Explosive growth phase, J. Geophys. Res., 97, 19311, 1992.

Ohtani, S., T. Higuchi, A. T. Y. Lui, and K. Takahashi, Magnetic fluctuations associated with tail current disruption: Fractal analysis, J. Geophys. Res., 100, 19135, 1995.

Roux, A., S. Perraut, A. Morane, P. Robert, A. Korth, G. .Kremser, A. Pederson, R. Pellinen, and Z. Y. Pu, Plasma sheet instability related to the westward traveling surge, J. Geophys. Res., 96, $17697,1991$.

Takahashi, K., L. J. Zanetti, R. E. Lopez, R. W. McEntire, T. A. Potemra, and K. Yumoto, Disruption of the magnetotail current sheet observed by AMPTE/CCE, Geophys. Res. Lett., 14, 1019, 1987.

This preprint was prepared with the AGU LATEX macros v3.0. File kbm paper formatted 1998 May 18.

With the extension package ' $\mathrm{AGU}^{++}$', version 1.2 from $1995 / 01 / 12$ 